\title{
Clumped stellar winds in supergiant high-mass X-ray binaries
}

\author{
L. M. Oskinova ${ }^{1}$, A. Feldmeier ${ }^{1}$, P. Kretschmar ${ }^{2}$ \\ ${ }^{1}$ Institute for Physics and Astronomy, University of Potsdam, 14476 Potsdam, Germany, \\ email: lida@astro.physik.uni-potsdam.de \\ ${ }^{2}$ European Space Astronomy Centre (ESA/ESAC), Science Operations Department, \\ Villanueva de la Cañada, Madrid, Spain
}

\begin{abstract}
The clumping of massive star winds is an established paradigm, which is confirmed by multiple lines of evidence and is supported by stellar wind theory. We use the results from time-dependent hydrodynamical models of the instability in the line-driven wind of a massive supergiant star to derive the time-dependent accretion rate on to a compact object in the Bondi-Hoyle-Lyttleton approximation. The strong density and velocity fluctuations in the wind result in strong variability of the synthetic X-ray light curves. Photoionization of inhomogeneous winds is different from the photoinization of smooth winds. The degree of ionization is affected by the wind clumping. The wind clumping must also be taken into account when comparing the observed and model spectra of the photoionized stellar wind.
\end{abstract}

Keywords. accretion, instabilities, stars: mass loss, X-rays: binaries

\section{Clumping of massive star winds}

Massive luminous OB-type stars possess strong stellar winds. The winds are fast, with typical velocities up to $2500 \mathrm{~km} \mathrm{~s}^{-1}$, and dense, with mass-loss rates $\dot{M} \gtrsim 10^{-7} M_{\odot} \mathrm{yr}^{-1}$. The driving mechanism for the mass-loss from OB stars has been identified with radiation pressure on spectral lines (Castor et al. 1975). Early on Lucy \& Solomon (1970) suggested that the stationary solution for a line-driven wind is unstable. Lucy \& White (1980) proposed that the winds break up into a population of dense blobs. Feldmeier et al. $(1997 \mathrm{a}, \mathrm{b})$ used hydrodynamic simulations to model the evolution of wind instabilities and found that the winds are non-stationary and inhomogeneous. The inhomogeneity affects stellar wind diagnostics. Stewart \& Fabian (1981) used Einstein spectra of O-stars to study the transfer of X-rays through a uniform stellar wind. They found that the massloss rate derived from X-ray spectra is lower by factor of a few than the mass-loss rates obtained from fitting the $\mathrm{H} \alpha$ line. As most plausible explanation for this discrepancy they suggested the neglect of the clumping in O-star winds. A theory of X-ray transfer in clumped winds that accounts for clumps of any shapes and optical depths was developed by Feldmeier et al. (2003) and Oskinova et al. (2004). Indeed, accounting for wind clumping allows to consistently model the UV/optical and the X-ray spectra of single O-stars (Oskinova et al. 2006, 2007). These conclusions are confirmed by full 3-D radiative-transfer models of stellar winds (Šurlan et al. 2012).

The mass-loss rates of massive stars are in good agreement with observed X-ray fluxes from high-mass X-ray binaries (HMXBs). HMXBs are the products of binary star evolution. These systems consist of an early-type massive star and a compact companion, neutron star (NS) or black hole (BH), on a close orbit (Iben et al. 1995). Accretion of stellar wind onto the compact companion powers the high X-ray luminosity of $\sim 10^{33}$ $10^{39} \mathrm{erg} \mathrm{s}^{-1}$. HMXBs can be divided in subclasses depending on the spectral type of the donor star - supergiant (SG) or Be-star. 


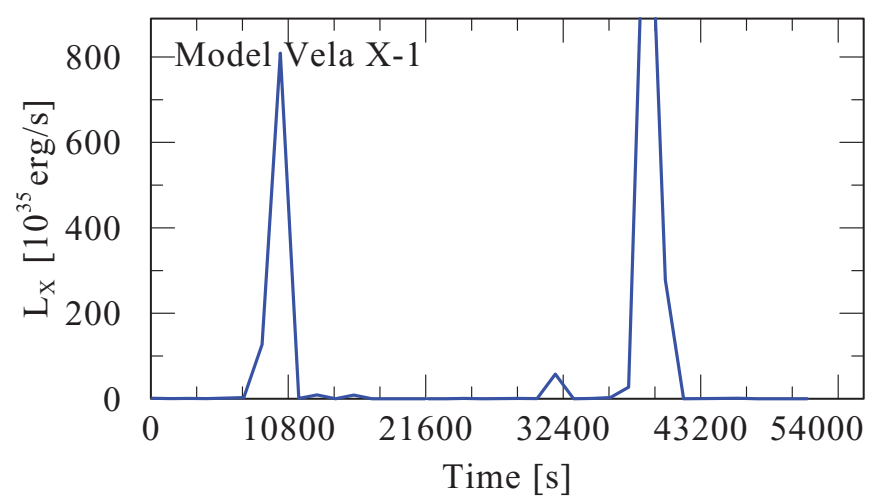

Figure 1. Synthetic X-ray light curves for the BondiHoyle accretion of a non-stationary stellar wind on to a NS with $m_{\mathrm{X}}=1.4 M_{\odot}$. Parameters of Vela X-1 were used in the simulation.

\section{Effect of wind-clumping for HMXBs}

We employ the results of hydrodynamical simulations of the line driven stellar wind presented in Feldmeier et al. (1997a,b). These hydrodynamic simulations derive the dynamic and thermal structure of stellar winds from the basic underlying physical principle, namely the acceleration of stellar wind by the line scattering of stellar UV photons.

The time dependent hydrodynamic simulations provide absolute values for the wind density and velocity as functions of radius and time. We use the predicted density and velocity field to compute synthetic X-ray light curves. An example of a light-curve is shown in Fig. 1. The variability is very large because of the strong variations in the wind velocity (shocks). The highly non-uniform wind density also contributes to the variability of accretion rate. The variations in the model light-curve are much larger than even strongest variability observed in HMXBs. We are prompted to conclude that simple Bondi-Hoyle accretion with clumped winds is not a sufficient explanation of variations in wind-accreting X-ray binaries.

Oskinova et al. (2012) showed that the photoionization of the wind by the radiation from accreting object is strongly affected by the wind clumping. In the optically thin case, the photoionization parameter has to be reduced by a factor $\chi$ compared to the smooth wind model, where $\chi$ is the wind inhomogeneity parameter.

Thus, wind clumping affects the accretion regime, the photoionization, and the wind opacity for the X-rays.

\section{References}

Castor, J. I., Abbott, D. C., \& Klein, R. I. 1975, ApJ, 195, 157

Feldmeier, A., et al. 1997a, A\&A, 320, 899

Feldmeier, A., Puls, J., \& Pauldrach, A. W. A. 1997b, A\& A, 322, 878

Feldmeier, A., Oskinova, L., \& Hamann, W.-R. 2003, A\&A, 403, 217

Iben, I., Jr, Tutukov, A. V., \& Yungelson, L. R. 1995, ApJS, 100, 217

Lucy, L. B. \& Solomon, P. M. 1970, ApJ, 159, 879

Lucy, L. B. \& White, R. L. 1980, ApJ, 241, 300

Oskinova, L. M., Feldmeier, A., \& Hamann, W.-R. 2004, A\& A, 422, 675

Oskinova L. M., Feldmeier A., Hamann W. 2006, MNRAS, 372, 313

Oskinova, L. M., Hamann, W., \& Feldmeier, A. 2007, A $\& A$, 476, 1331

Oskinova, L. M., Feldmeier, A., \& Kretschmar, P. 2012, MNRAS, 421, 2820

Stewart, G. C. \& Fabian, A. C. 1981, MNRAS, 197, 713

Šurlan, B. et al. 2012, A\&A, 541, 37 